This article was downloaded by: [Stewart, Phil]

On: 28 January 2011

Access details: Access Details: [subscription number 932852886]

Publisher Taylor \& Francis

Informa Ltd Registered in England and Wales Registered Number: 1072954 Registered office: Mortimer House, 3741 Mortimer Street, London W1T 3JH, UK

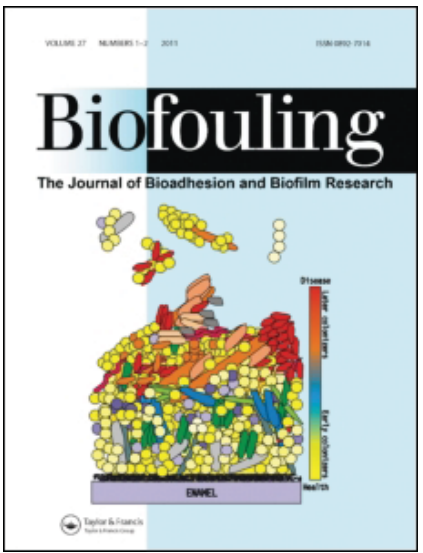

\title{
Biofouling
}

Publication details, including instructions for authors and subscription information:

http://www.informaworld.com/smpp/title $\sim$ content=t713454511

\section{Chemical and antimicrobial treatments change the viscoelastic properties of bacterial biofilms}

Warren L. Jones ${ }^{\mathrm{a}}$; Michael P. Sutton ${ }^{\mathrm{b}}$; Ladean McKittrick ${ }^{\mathrm{a}}$; Philip S. Stewart

${ }^{a}$ Department of Civil Engineering, Montana State University, Bozeman, Montana, USA ${ }^{\mathrm{b}}$ Anadarko

Petroleum, WY, USA ${ }^{c}$ Center for Biofilm Engineering, Montana State University, Bozeman, Montana, USA

First published on: 27 January 2011

To cite this Article Jones, Warren L. , Sutton, Michael P. , McKittrick, Ladean and Stewart, Philip S.(2011) 'Chemical and antimicrobial treatments change the viscoelastic properties of bacterial biofilms', Biofouling, 27: 2, $207-215$, First published on: 27 January 2011 (iFirst)

To link to this Article: DOI: $10.1080 / 08927014.2011 .554977$

URL: http://dx.doi.org/10.1080/08927014.2011.554977

\section{PLEASE SCROLL DOWN FOR ARTICLE}

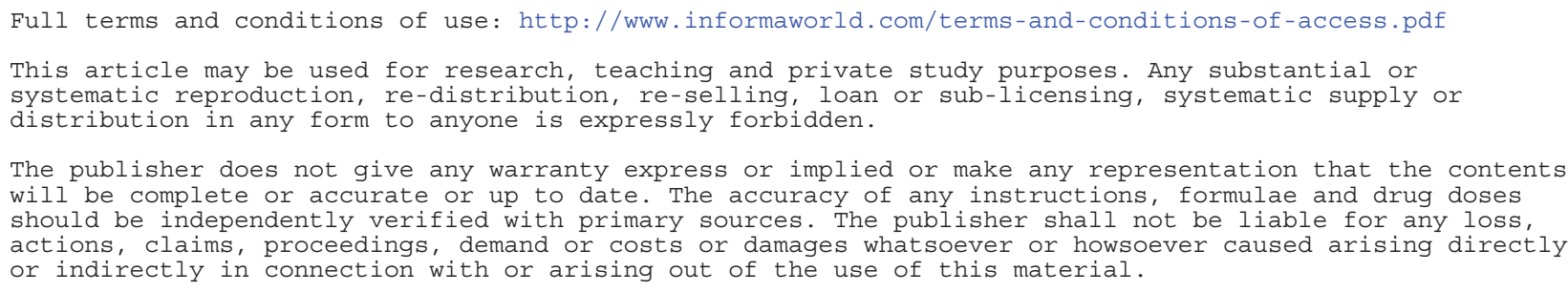




\title{
Chemical and antimicrobial treatments change the viscoelastic properties of bacterial biofilms
}

\author{
Warren L. Jones ${ }^{\mathrm{a} *}$, Michael P. Sutton ${ }^{\mathrm{b}}$, Ladean McKittrick ${ }^{\mathrm{c}}$ and Philip S. Stewart ${ }^{\mathrm{d}}$ \\ ${ }^{a}$ Department of Civil Engineering, Montana State University, Bozeman, Montana, USA; ${ }^{b}$ Anadarko Petroleum, 1400 E Lincoln \\ Gillette, WY 82717, USA; ${ }^{c}$ Department of Civil Engineering, Montana State University, Bozeman, Montana, USA; ${ }^{d}$ Center for \\ Biofilm Engineering, Montana State University, Bozeman, Montana, USA
}

(Received 27 October 2010; final version received 9 January 2011)

\begin{abstract}
Changes in the viscoelastic material properties of bacterial biofilms resulting from chemical and antimicrobial treatments were measured by rheometry. Colony biofilms of Staphylococcus epidermidis or a mucoid Pseudomonas aeruginosa were subjected to a classical creep test performed using a parallel plate rheometer. Data were fit to the 4-parameter Burger model to quantify the material properties. Biofilms were exposed to the chloride salts of several common mono-, di-, and tri- valent cations, and to urea, industrial biocides, and antibiotics. Many of these treatments resulted in statistically significant alterations in the material properties of the biofilm. Multivalent cations stiffened the $P$. aeruginosa biofilm, while ciprofloxacin and glutaraldehyde weakened it. Urea, rifampin, and a quaternary ammonium biocide weakened the $S$. epidermidis biofilm. In general, there was no correspondence between the responses of the two different types of biofilms to a particular treatment. These results underscore the distinction between the killing power of an antimicrobial agent and its ability to alter biofilm mechanical properties and thereby influence biofilm removal. Understanding biofilm rheology and how it is affected by chemical treatment could lead to improvements in biofilm control.
\end{abstract}

Keywords: biofilm; viscoelasticity; Burger model; cation; antimicrobial; rheometry

\section{Introduction}

Biofouling is a costly and sometimes deadly problem facing industry and medicine. Millions of dollars in extra costs occurring from pressure losses in pipe systems, increased drag on ships' hulls, down time for cleaning and part replacements are blamed on biofouling every year. Pathogens may be introduced into drinking water systems through biofilm detachment (Van der Wende et al. 1989). Persistent infections formed on implanted medical devices are one of the greatest obstacles currently facing the medical industry (Costerton et al. 1999; Habash and Reid 1999). The main culprits for biofouling are biofilms. Antimicrobial agents are the main chemical weapon used to control biofilms. Antimicrobials work by stopping or slowing microbial growth, but do not necessarily clear the biofilms from the affected area. Much more is known about killing biofilm than is known about removing biofilm (Chen and Stewart 1996).

The discovery and development of technologies for dispersing microbes from biofilms rather than killing them presents an opportunity for controlling detrimental biofilms. Dispersal strategies have a few attractive features. Microorganisms in biofilms are known to be protected from killing by antimicrobial agents (Costerton et al. 1999), so alternative approaches that bypass these defenses are needed. Because most antimicrobial agents are inherently toxic, dispersal technologies have the potential to be more environmentally benign. In many real-world applications, a reduction in fouling rather than a reduction in viability is the primary desired outcome. This is true, for example, in such diverse contexts as ship hull fouling and urinary catheter encrustation. A paradigm shift from killing to removal motivates inquiry into: (1) the composition of the biofilm matrix and the mechanisms of matrix cohesion, (2) the natural pathways of biofilm dispersal and detachment, and (3) experimental and theoretical methods for characterizing the material and failure properties of biofilms.

Whether applying an antimicrobial agent or a chemical treatment with dispersing capability, it is important to understand how the treatment affects the material properties and cohesion of biofilms. If the goal is to remove the biofilm from a surface then choosing a treatment that weakens the biofilm would most likely be the best choice. If the goal is to prevent the dispersal of many cells from a biofilm into a system, then using a treatment that makes the biofilm more cohesive may be a better choice. The ability to

*Corresponding author. Email: warren_j@biofilm.montana.edu

Published online 27 January 2011

ISSN 0892-7014 print/ISSN 1029-2454 online

(C) 2011 Taylor \& Francis

DOI: $10.1080 / 08927014.2011 .554977$

http://www.informaworld.com 
manipulate the material properties of biofilm may prove a valuable tool in controlling biofilm formation and removal.

In this investigation, the authors sought to develop and apply protocols to quantify changes in the material properties of biofilms in response to a wide variety of treatments. Prior work on the mechanical properties of biofilms provides insight and guidance on how to best do this. Earlier work on creating mechanical models of biofilms was performed using microscopy in fluid flow cells where the deformation of a biofilm cluster was measured under a known flow rate. From the flow rate, a shear stress could be calculated and from the microscopy a strain and strain rate could be measured. These tests indicated that the biofilm could be modeled as a viscoelastic fluid (Klapper et al. 2002; Stoodley et al. 2002). Alteration of the mechanical properties of biofilm has been explored by Chen and Stewart (2002), Klapper et al. (2002), Towler et al. (2003), and Wloka et al. (2005). Work on determining the cohesive strength of biofilm flocs has been performed using a micro-cantilever technique (Poppele and Hozalski 2003). The force required to separate a biofilm floc was determined by microscopically measuring the deflection of a small glass pipette tip at the mechanical failure of the biofilm sample, and the ultimate stress was determined by dividing the force by the estimated area of the failure plane. Subsequent work (Aggarwal and Hozalski 2010; Aggarwal et al. 2010) on Staphylococcus epidermidis and Pseudomonas aeruginosa biofilms utilized video image analysis to also develop estimates of the elastic modulus, failure strain and toughness, and examined issues of colony size, measurement scale and contact angle of the microcantilever. Uniaxial compression techniques have been used to help estimate the mechanical properties of biofilms. The apparent modulus of elasticity and yield point can be estimated using this method (Korstgens et al. 2001). Hohne et al. (2009) used a microfluidic device to induce a measurable stress on a biofilm attached to a membrane, and measured the resulting strain with a confocal scanning laser microscope; a similar process using a microindentation system was employed by Cense et al. (2006). One of the most microscale techniques was employed by Lau et al. (2009) in which an atomic force microscope was used in conjunction with microbeads to evaluate forces on cells at the individual cellular scale. In their work with wild type and LPS mutant strains of $P$. aeruginosa PAO1, adhesive pressures (equivalent to a tensile shear strength) were found to be significantly higher than the shear stress employed in the current investigation.

Little research has focused on altering the mechanical properties of biofilms (Stoodley et al. 2002;
Wloka et al. 2005). The effects of chemical treatments on the cohesion of biofilm to surfaces were investigated (Chen and Stewart 2000). This investigation measured the protein released from biofilm on a stainless steel slide by chemical treatments, and correlated protein removal to biofilm removal. The effect of a chemical on the detachment rate of a biofilm was determined. The addition of calcium ions to the EPS was shown by Chen and Stewart (2002) to affect its overall stiffness.

The purpose of the work reported in this article was to demonstrate that a wide variety of outcomes are possible in terms of changes to the material properties of biofilms when treated with an antimicrobial agent or potential dispersing agent. It is not possible to know a priori whether a particular treatment will have no effect, a weakening effect, or a strengthening effect. The data suggest that educated guesses or assumptions based on current conceptual models may be unreliable. For example, it is shown that exposure to calcium does not always enhance biofilm cohesion, that exposure to glutaraldehyde can weaken a biofilm rather than crosslink it as expected, and that antibiotics can substantially reduce biofilm cohesion even though these agents are thought of as specifically cidal in their action.

\section{Modeling}

The Burger model of viscoelastic behavior was used to interpret experimental results (Towler et al. 2007). This model combines elastic and inelastic discrete elements which can be represented as springs and dashpots, respectively (Figure 1). To capture the phenomena contained in an experimental creep curve, these elements are combined in parallel (the Kelvin model, denoted by the subscript $\mathrm{K}$ ) and a second spring and dashpot are added in series (the Maxwell model, denoted by the subscript $\mathrm{M}$ ). The constitutive equation relating stress and strain for the spring element is Hooke's Law for elastic solid materials. Newton's law of viscosity for viscous fluids relates stress and strain rate for the dashpot elements. Both of these relationships are linear, so that combinations of these elements will also respond in a linear fashion.

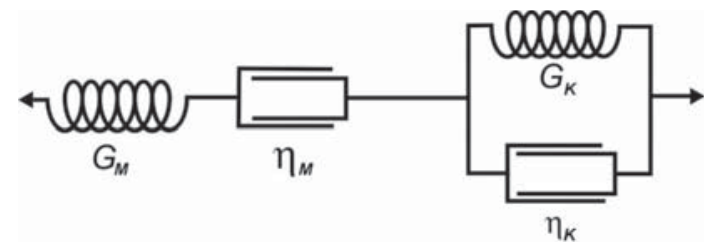

Figure 1. Schematic representation of the combined parallel and series elements constituting the Burger model for viscoelastic materials. 
When elements are combined in series, all elements are subjected to the same stress, and are free to strain in response to that stress; the sum of the strains is the total for the construct. When elements are combined in parallel, they both experience the same strain, and the stress experienced by each is summed to give the total stress.

In the creep test conditions, with zero strain at time zero, an imposed stress, $\tau$, is added at $t=0$ and applied for $t_{1}$ seconds $(300 \mathrm{~s}$ or $5 \mathrm{~min}$ in these experiments). At $t=t_{1}$, the stress is removed $(\tau=0)$, so that a new initial condition is developed with a nonzero initial strain, and the construct is allowed to "relax" for an additional 5 min. The development of the final response of the system to the added and then released stress produces an unique analytical solution to the strain curve for each of these time periods as follows. For the time period $0<t<t_{1}$, the strain $(\gamma)$ is given by:

$$
\gamma(t)=\frac{\tau}{G_{M}}+\frac{\tau}{\eta_{M}} t+\frac{\tau}{G_{K}}\left(1-\exp \left(-\frac{G_{K}}{\eta_{K}} t\right)\right)
$$

where $G_{M}$ and $G_{K}$ are the Maxwell and Kelvin spring constants, respectively $(\mathrm{kPa})$, and $\eta_{M}$ and $\eta_{K}$ are the Maxwell and Kelvin dashpot constants or viscosities, respectively $\left(\mathrm{kPa} \mathrm{s}^{-1}\right)$. Once the stress is removed at $t_{1}$, the Maxwell elastic element returns to a strain of zero, the Maxwell viscous element remains at the same strain it experienced at $t=t_{1}$, and the Kelvin construct experiences a net zero stress. As a result, for $t>t_{1}$, the total strain is given by:

$$
\gamma(t)=\frac{\tau}{\eta_{M}} t_{1}+\frac{\tau}{G_{K}}\left(1-\exp \left(-\frac{G_{K}}{\eta_{K}} t_{1}\right)\right) \exp \left(-\frac{G_{K}}{\eta_{K}} t\right)
$$

A typical result from a creep test is shown in Figure 2 (experimental and fitted curve), along with labels showing which portions of the curve are governed by particular parameters. At $t=0$, the dashpot in the parallel (Kelvin) model prevents any initial strain, but the spring in the series portion can instantaneously strain to its ultimate length. Thus, the initial jump in the creep curve is a function of the spring constant $G_{M}$. Once the Kelvin element is stretched so that the stress in it is carried all by the spring, the overall strain reflects only the character of the Maxwell dashpot, $\eta_{M}$. After the stress is removed, the strain should drop immediately by the same value that it jumped at $t=0$, reflecting the relaxation of the Maxwell spring, and then the strain will continue to decrease as the stored stress in the Kelvin spring is released through its parallel dashpot. At equilibrium, there is a net displacement reflecting the effects of the

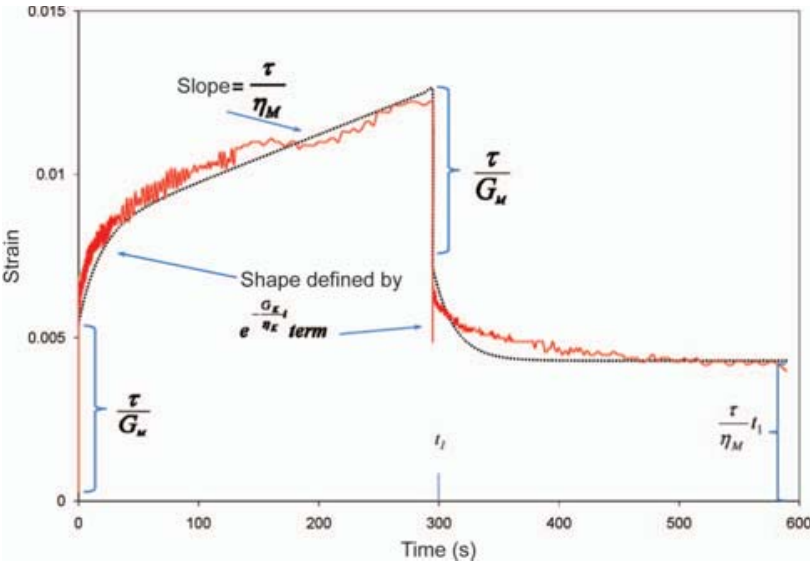

Figure 2. Typical creep curve response showing regions influenced by each of the Burger parameters. Stress is applied for the first $300 \mathrm{~s}$ and removed at $t=300 \mathrm{~s}$, while strain is monitored for an additional $300 \mathrm{~s}$ under zero applied shear. The smooth curve is the fitted (model), while the more erratic curve represents the actual data set (FRD1 with $0.2 \mathrm{M} \mathrm{CaCl}_{2}$ treatment).

Maxwell dashpot, and the regions of the curves between the points described above reflect the relative importance of the Kelvin elements. Note that without any one of the four elements used in this model, the key features of the creep curve would not be captured by the resulting model, regardless of the parameter values.

\section{Methods}

\section{Bacterial strains}

Creep testing was performed on colony biofilms of two distinct species of bacteria. The first, $P$. aeruginosa, is a Gram-negative aerobic organism that has been extensively studied due to its implication in morbidity and mortality associated with cystic fibrosis (Silo-Suh et al. 2002; Walters et al. 2003). The strain used in this study is FRD1, a mucoid phenotype that overexpresses the exopolysaccharide alginate. The second organism was $S$. epidermidis, a Gram-positive coccoid facultative anaerobe commonly found as part of the natural flora of skin. S. epidermidis is also implicated as an opportunistic pathogen, particularly associated with nosocomial infections associated with catheters and implants (Rupp et al. 1999). The EPS of S. epidermidis is known to contain polyglucosamine and teichoic acid moieties (Sadovskaya et al. 2005). The strain of $S$. epidermidis used in this study was ATCC strain \#35984.

\section{Biofilm growth and treatment}

Biofilms were grown using the colony biofilm method (Anderl et al. 2000; Walters et al. 2003). This technique 
has been shown to produce a morphology that is both physically and physiologically similar to biofilms grown under fluid shear while submerged, and while it cannot be said to exactly match a "normal" biofilm, the technique allows rapid and reproducible generation of samples for testing. An overnight culture grown on tryptic-soy broth (TSB) was diluted to an absorbance of 0.05 at $600 \mathrm{~nm}$. Polycarbonate filter membranes ( $25 \mathrm{~mm}, 0.2 \mu \mathrm{m}$ pore size) that had been UV-sterilized (10 min each side) were used as the substratum on which to grow the biofilm. Sterilized membranes were placed onto a tryptic-soy agar (TSA) plate, and a drop of $0.3 \mathrm{ml}$ of the diluted overnight culture was then pipetted onto the center of each of the filter membranes, and then carefully spread over the entire area of the membrane without spilling the inoculum over the edge. The inoculated membranes were then allowed to grow in an incubator at $37^{\circ} \mathrm{C}$ for $24 \mathrm{~h}$ before testing. Over this period of time, a colony $80-150 \mu \mathrm{m}$ in thickness developed on the membrane.

With two exceptions, treatments of test samples were performed by pipetting $0.47 \mathrm{ml}$ of the desired treatment onto a $25 \mathrm{~mm}$ filter pad, then placing the membrane containing the developed colony on top of the filter pad for $1 \mathrm{~h}$, allowing the treatment solution to diffuse into the biofilm. Following the treatment, the membrane was moved directly to the rheometer for creep testing. The two exceptions to the treatment regime described above were the treatments with antibiotics. When S. epidermidis biofilms were treated with rifampin, the treatment was accomplished by moving the membrane containing a mature (24-h) biofilm to a TSA plate containing $0.1 \mu \mathrm{g} \mathrm{ml}^{-1}$ rifampin for $24 \mathrm{~h}$ prior to testing. Similarly, the ciprofloxacin treatment of mature (24-h) FRD1 biofilms was done by moving the membrane to TSA plates containing $1.0 \mu \mathrm{g} \mathrm{ml}^{-1}$ of ciprofloxacin for $24 \mathrm{~h}$ before testing.

A detailed list of all treatments and concentrations is presented in as part of the results (Table 1). Chloride salts of the metal cations ( $\mathrm{Na}, \mathrm{Ca}, \mathrm{Mg}, \mathrm{Al}, \mathrm{Fe}(\mathrm{II})$, and $\mathrm{Fe}(\mathrm{III}))$ were purchased from Fisher Scientific. Glutaraldehyde, quaternary ammonium compound (QAC), urea, and EDTA (ethylene diamine tri-acetic acid, disodium salt) and rifampin and ciprofloxacin were purchased from Sigma, while ATMPA (amino tri(methylene-phosphonic acid), pentasodium salt) was purchased as Dequest 2006 from Solutia. Chlorine was prepared by dilution of a titrated solution of commercial bleach to the desired concentration.

Table 1. Test conditions and median values of the fitted Burger parameters, the strain at the peak of the creep curve, and the number of biofilms tested $(n)$ under each condition.

\begin{tabular}{|c|c|c|c|c|c|c|c|c|}
\hline Organism & Treatment & $\begin{array}{c}G_{K} \\
(\mathrm{kPa})\end{array}$ & $\begin{array}{c}\eta_{K} \\
\text { (kPa.s) }\end{array}$ & $\begin{array}{c}\eta_{M} \\
\text { (kPa.s) }\end{array}$ & $\begin{array}{c}G_{M} \\
(\mathrm{kPa})\end{array}$ & $\begin{array}{c}\text { Peak } \\
\text { strain }(-)\end{array}$ & $\begin{array}{l}\text { Gap } \\
(\mu \mathrm{m})\end{array}$ & $n$ \\
\hline \multirow[t]{17}{*}{ P. aeruginosa FRD1 } & Control & 0.43 & 11 & 40 & 1.1 & 0.16 & 135 & 153 \\
\hline & $\mathrm{NaCl}, 0.2 \mathrm{M}$ & 0.47 & 21 & 50 & 2.3 & 0.13 & 134 & 16 \\
\hline & $\mathrm{CaCl}_{2}, 0.2 \mathrm{M}$ & 1.40 & 34 & 230 & 1.4 & 0.041 & 111 & 18 \\
\hline & $\mathrm{MgCl}_{2}, 0.2 \mathrm{M}$ & 0.80 & 21 & 110 & 2.8 & 0.06 & 87 & 18 \\
\hline & $\mathrm{FeCl}_{2}, 0.02 \mathrm{M}$ & 0.97 & 30 & 140 & 1.3 & 0.053 & 73 & 11 \\
\hline & $\mathrm{FeCl}_{2}, 0.2 \mathrm{M}$ & 3.10 & 141 & 500 & 3.2 & 0.018 & 98 & 20 \\
\hline & $\mathrm{AlCl}_{3}, 0.02 \mathrm{M}$ & 1.06 & 22 & 130 & 1.2 & 0.061 & 92 & 14 \\
\hline & $\mathrm{AlCl}_{3}, 0.2 \mathrm{M}$ & 1.50 & 48 & 420 & 2.9 & 0.027 & 111 & 24 \\
\hline & $\mathrm{FeCl}_{3}, 0.02 \mathrm{M}$ & 1.60 & 21 & 220 & 1.9 & 0.036 & 95 & 22 \\
\hline & $\mathrm{FeCl}_{3}, 0.2 \mathrm{M}$ & 10.50 & 176 & 1971 & 9.9 & 0.006 & 112 & 15 \\
\hline & EDTA, $0.2 \mathrm{M}$ & 0.47 & 12 & 7.2 & 0.3 & 0.78 & 129 & 9 \\
\hline & ATMPA, $1.0 \mathrm{~g} \mathrm{l}^{-1}$ & 1.20 & 62 & 140 & 1.2 & 0.056 & 104 & 19 \\
\hline & Chlorine, $50 \mathrm{mg} \mathrm{l}^{-1}$ & 0.27 & 6.5 & 20 & 1.6 & 0.28 & 184 & 14 \\
\hline & Urea, $0.2 \mathrm{M}$ & 0.19 & 8.9 & 8.8 & 0.8 & 0.61 & 163 & 19 \\
\hline & QAC, $50 \mathrm{mg}^{-1}$ & 0.03 & 6.3 & 4.1 & 0.7 & 1.25 & 170 & 22 \\
\hline & Glutaraldehyde, $50 \mathrm{mg}^{-1}$ & 0.70 & 2.4 & 3.1 & 0.8 & 1.63 & 157 & 10 \\
\hline & Ciprofloxacin, $1 \mathrm{mg} \mathrm{l}^{-1}$ & 0.07 & 135 & 0.8 & 0.5 & 6.2 & 101 & 10 \\
\hline \multirow[t]{9}{*}{ S. epidermidis } & Control & 28 & 775 & 3900 & 10 & 0.02 & 82 & 39 \\
\hline & $\mathrm{NaCl}, 0.2 \mathrm{M}$ & 7.8 & 150 & 830 & 3.4 & 0.071 & 80 & 7 \\
\hline & $\mathrm{CaCl}_{2}, 0.2 \mathrm{M}$ & 9 & 230 & 950 & 6 & 0.063 & 74 & 9 \\
\hline & $\mathrm{FeCl}_{3}, 0.2 \mathrm{M}$ & 16 & 920 & 2600 & 9.3 & 0.028 & 62 & 9 \\
\hline & EDTA, $0.2 \mathrm{M}$ & 29 & 750 & 3000 & 9 & 0.025 & 73 & 8 \\
\hline & Urea, $0.2 \mathrm{M}$ & 2.1 & 58 & 92 & 0.6 & 0.52 & 83 & 6 \\
\hline & QAC, $50 \mathrm{mg}^{-1}$ & 2.6 & 31 & 140 & 1.5 & 0.29 & 73 & 4 \\
\hline & Glutaraldehyde, $50 \mathrm{mg}^{-1}$ & 41 & 720 & 6200 & 7.4 & 0.021 & 97 & 9 \\
\hline & Rifampin, $0.1 \mathrm{mg} \mathrm{l}^{-1}$ & 4.2 & 42 & 400 & 3.4 & 0.11 & 55 & 9 \\
\hline
\end{tabular}

Values in bold indicate significant difference from the control at the $p<0.05$ level. 


\section{Test apparatus}

A TA Instruments AR1000 rheometer was used for all rheological testing (Towler et al. 2003). The AR 1000 is a disk type rheometer in which a sample is placed between a base plate and a cylindrical head that can be lowered into position for testing. A $25 \mathrm{~mm}$ aluminum base plate was used in all tests. The biofilm membranes were positioned directly in the center of the base plate so that the cylindrical head completely covered the biofilm sample, prewetted to ensure complete submersion of the face of the rheometer head contacting the biofilm. A humidification chamber was also developed so that the sample did not lose mass due to drying during the tests, which lasted $<15 \mathrm{~min}$ from loading to disassembly.

\section{Creep test protocol}

Consistent and reproducible results with the rheometer rely on uniform contact between the head of the rheometer and the biofilm. Because the biofilms were not all of uniform thickness, particularly after treatment, gap setting alone was an unreliable means of ensuring complete contact between the head and sample. Once the head contacted the biofilm, however, lowering the head by a small interval produced an initial normal force that was observed to relax to zero over the ensuing few seconds. By lowering the head in $5 \mu \mathrm{m}$ intervals, and allowing the normal force to dissipate between intervals, a curve of normal force $v s$ gap width was developed. The normal force vs gap curve should be linear for materials following Hooke's Law. The normal force at which this relationship of distance vs force became linear was determined to be $1.0-1.5 \mathrm{~N}$ for the FRD1 biofilm, while it was $0.5 \mathrm{~N}$ for the $S$. epidermidis. Mean normal force $v s$ gap data are shown in Figure 3. Thus, gap was set according to normal force rather than a specific distance.

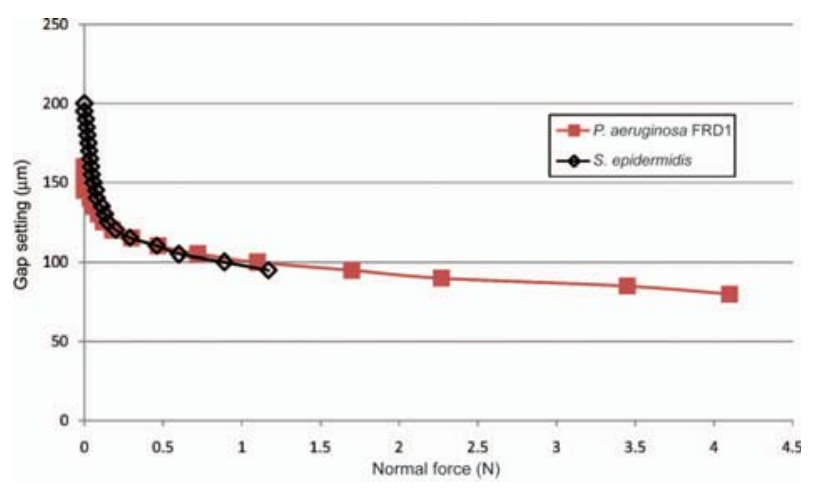

Figure 3. Instantaneous normal force on the rheometer head as the gap setting is decreased (control biofilms of each species).
The next assumption implicit in the development of the Burger model is that the viscoelastic behavior of the materials is linear. For the FRD1 biofilm, a series of creep tests were run at shear stresses between $10 \mathrm{~Pa}$ and $30 \mathrm{~Pa}$. For each of these, the compliance was computed (compliance is the strain divided by the stress; it is a measure of stiffness). The compliance should be the same for all tests if the material is linear. This occurred at about $15 \mathrm{~Pa}$ for the $P$. aeruginosa FRD1 biofilm. With the $S$. epidermidis biofilm, no measurable strain resulted at this low shear stress. At a shear stress of $100 \mathrm{~Pa}$, the peak strain was large enough to distinguish among treatments and was still in the linear range, so this stress value was used for the S. epidermidis biofilm.

In the performance of the creep tests, after setting the gap as described above, shear stress at the predetermined level described above was applied for exactly $5 \mathrm{~min}$, followed by a 5 -min period with zero applied shear. During this period, $\sim 970$ data points for the strain experienced by the head were taken and recorded by the computer system. Each biofilm sample was tested only once. Replicate samples (up to 17, in the case of some controls) were performed on replicate membrane/biofilm samples. Control tests with filters alone (no biofilm) were performed, and it was concluded that filters did not move relative to the fixed base during the applied shear.

\section{Parameter estimation}

Assuming the biofilm tested in the creep experiments responded in a nearly linear-viscoelastic manner, the four material coefficients from the Burger's model equations ( 1 and 2) can be numerically optimized to fit the data. Non-linear regression techniques would be the preferred method for optimizing the four material coefficients; however, the nature of creep tests makes gradient methods impossible. The applied shear stress during creep testing experiences a step change that occurs between the loading and unloading phases of the test. The step change in applied shear stress causes a discontinuity in the derivatives of the equations describing material behavior. The discontinuity of the derivatives prohibits many analytical methods that employ calculus based optimization techniques. A least-square error technique was developed for solution using the built-in solver in Microsoft Excel. The error function was minimized with respect to each of the four parameters individually in turn and then globally with respect to all four until successive iterations of these 5 steps resulted in no change in the parameter values (relative change $<10^{-8}$ ).

Time and shear strain data from the creep test experiments were not taken in evenly spaced time 
intervals. The sampling rates from the creep tests were varied from every $0.0016 \mathrm{~s}$ to $6.5 \mathrm{~s}$, as generated by the rheological software in response to the rate of change in the strain values. To ensure relatively equal fitting rigor over the entire time course of the test, each error value was multiplied by the time lapse since the last data point, divided by the entire test time. The overall function minimized in finding the Burger parameters is thus given by:

$$
\operatorname{MSE}=\sum_{i}\left(\left(\gamma_{i}-\gamma_{\mathrm{fit}, i}\right)\left(\frac{\Delta t_{i}}{t_{\mathrm{total}}}\right)\right)^{2} / n
$$

where $\gamma_{i}$ is the measured strain value at time $t_{i}, \gamma_{\mathrm{fit}, i}$ is the strain value predicted by the Burger model at that time point, $\Delta t_{i}$ is the sample interval at time $t_{i}$ and $n$ is the total number of data points in that run. With this weighting function applied, the curve fit avoids excessive weighting in the steeper portions of the creep curve, and provides a better fit in the flat portions where the slope and intercept correlate strongly with the coefficient values.

\section{Statistical analyses}

The values of the Burger coefficients and the peak strain value did not follow a normal distribution for any of the treatment or control conditions. A logarithmic transformation of the variables did produce a relatively normal distribution, so all further analyses were performed on log-transformed parameters. Pairwise $t$-tests assuming unequal variance were used to compare each of the Burger parameters for the treated biofilms with the parameters for the corresponding untreated controls. Differences were taken as significant for $p<0.05$. Minitab ${ }^{\circledR}$ statistical software was used to perform all two-variable $t$-tests.

\section{Results}

A typical creep curve is shown in Figure 2, where direct data collected during a creep test are overlaid with the regression curve obtained from the fitting of the data to the Burger model. Examples of the regression curves obtained from multiple tests of both species of biofilm with and without urea treatment are presented in Figure 4. Comparison of these two figures reveals some of the kinds of trends observed in this study. First, the FRD1 biofilm exhibited much larger strains than the S. epidermidis biofilm, which becomes even more significant when a comparison is made with the shear stress used to produce these strains ( $15 \mathrm{~Pa} v s 100 \mathrm{~Pa}$, respectively). Secondly, the effect of the urea on both biofilms was to produce larger strains, but the effect was more
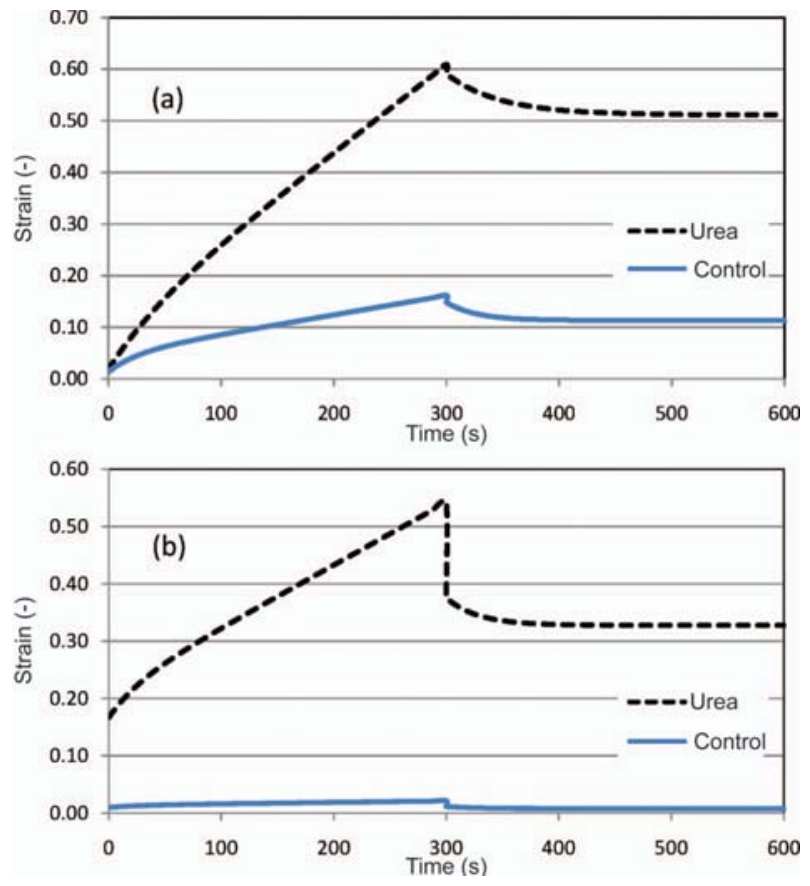

Figure 4. Typical creep curves from fitted parameter values. (a) = response of untreated $\left(-\right.$ ) and urea treated $(--)^{-}$ biofilms of $P$. aeruginosa FRD1; (b) $=$ response of untreated ( - ) and urea treated (- - ) biofilms of S. epidermidis.

pronounced on the $S$. epidermidis biofilm than on the FRD1 biofilm.

Values for the Burger parameters, peak strain (the peak value at the end of the applied stress period) and gap setting are shown in Table 1. All four of the Burger parameters are significantly higher for the untreated S. epidermidis biofilm than for the untreated $P$. aeruginosa FRD1 biofilm, as is reflected in the necessity of applying a higher shear stress to the S. epidermidis biofilm. Without treatment, and grown on the same medium, the $S$. epidermidis biofilm was 8 to almost 100 times stiffer (modulus of elasticity, $G$ ) and more viscous (viscosity, $\eta$ ).

A compendium of all of the test and treatment effects is presented in Table 1. In this table, each of the treatments (and controls) of both biofilms are listed. The values of the four Burger parameters obtained from the fitting of the creep curves are also presented, along with the peak strain (the mean height of the peak on the creep curve) and the mean gap used. The number of biofilms tested is also included. Parameter values in bold face for the treated biofilms indicate that the value is significantly different at the 0.05 level from the untreated control. Values of standard error (SE) or variance are not included in Table 1 for the sake of brevity. The coefficient of variation (on the logarithmic scale used for statistical analyses) was $5-20 \%$ for the 
FRD1 control sample Burger parameters, and 3-8\% for the S. epidermidis control sample Burger parameters. The coefficients of variation for the treated samples were similar for treatments that produced a stiffer or more viscous biofilm, but much higher when the biofilm was weakened significantly (eg the CV values for the ciprofloxacin treatment of FRD1 were $25 \%$ to $70 \%$, and for urea were $>40 \%$ for both species).

Several patterns emerge from the data. With regard to occurrence and patterns of significance, it appears that most of the treatments that produced a significant change in one parameter produced a significant change in all of them, particularly the multivalent metals with FRD1. Treatments that reduced the Burger parameter values tended to also produce higher peak strain values. Treatments that decreased the peak strain also were observed to begin with smaller gap settings. Conversely, smaller gap settings were not universally associated with lower peak strains. Burger parameters tended to all move in the same direction with FRD1, while with $S$. epidermidis, a single treatment might produce an increase in some parameters, decreases in others, and result in no change in the peak strain.

\section{Discussion}

Despite having similar qualitative responses to compressive forces (Figure 3), biofilms of S. epidermidis and $P$. aeruginosa clearly showed different responses to shear (Table 1). All four of the Burger parameters were significantly higher for the S. epidermidis than for the $P$. aeruginosa biofilm, and the peak strain value was likewise significantly smaller for the $S$. epidermidis. To put the values of the Burger parameters in context, the two viscosity parameters were of the order of $10^{6} \mathrm{~Pa}-\mathrm{s}$ for the $S$. epidermidis biofilms and of the order of 20,000 Pa-s for the $P$. aeruginosa biofilm. The shear moduli for the two biofilms were on the order of 15,000 and $500 \mathrm{~Pa}$ for the S. epidermidis and P. aeruginosa, respectively. Generally, a description of biofilm based solely on these measurements would place it somewhere between a highly viscous fluid and a very elastic solid than either type of material by itself. These observations are consistent with those of Stoodley et al. (2002) and Towler et al. (2003), who observed deformation behavior of biofilms and found both fluid and solid characteristics. The $S$. epidermidis shear moduli in this study were about 5 times higher than measured by Hohne et al (2009), but in the same order of magnitude.

As was shown in Table 1, the values of the Burger parameters as well as the value of the peak strain varied widely in response to the treatment of the biofilms, with some treatments producing order-of- magnitude changes in biofilm properties. To better visualize the effect of the treatments, the ratio of the median peak strain for a treated biofilm to the median peak strain for an untreated biofilm was determined for all of the treatments. These are shown in Figure 5 (P. aeruginosa) and Figure 6 (S. epidermidis). Gray bars indicate a peak strain ratio that was determined by a pairwise $t$-test to be significantly different from 1.0 at the 0.05 significance level, while black bars indicate a significant difference from the control at the 0.01 level.

It is interesting to note that the multivalent cation treatments of the $P$. aeruginosa biofilm produced decreases in the strain (and, referring to Table 1, increases in most of the Burger parameters) in all cases.

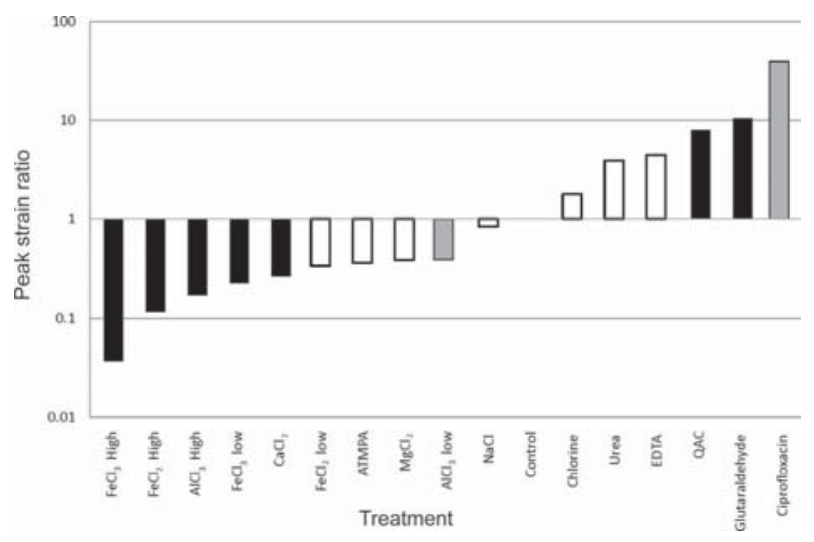

Figure 5. Treated $P$. aeruginosa FRD1 biofilm peak strain values normalized to the peak strain of the control biofilm. indicates significantly different values from 1.0 at the 0.01 level, while indicates significance at $0.01<p<0.05$.

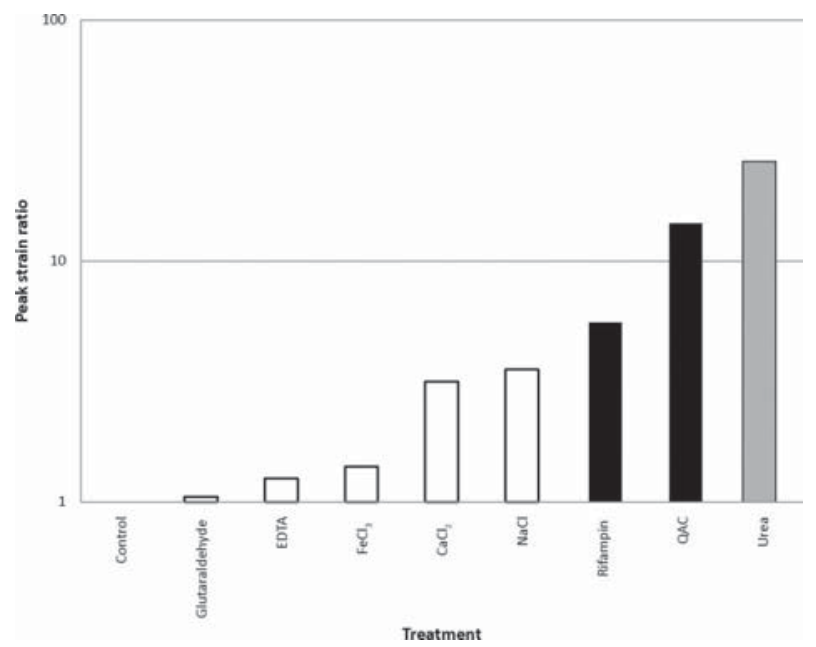

Figure 6. Treated S. epidermidis biofilm peak strain values normalized to the peak strain of the control biofilm. indicates significantly different values from 1.0 at the 0.01 level, while $\square$ indicates significance at $0.01<p<0.05$. 
Iron produced the strongest effect of all the cations, and higher concentrations (where more than one was used) produced more effect than lower concentrations. This result was, to some degree, anticipated based on the production of alginate by the FRD1 strain of $P$. aeruginosa. The presence of multiple carboxylate groups along the alginate molecule would suggest many sites for binding and cross-linking of molecules by multivalent cations. The addition of a monoprotic cation, $\mathrm{Na}^{+}$, appeared to have little effect, and the addition of EDTA as a multivalent cation chelator produced only moderate increases in strain, while the addition of ATMPA produced a decrease in the peak strain and increases in the Burger parameters. In contrast, the addition of multivalent cations to the $S$. epidermidis biofilms produced comparatively little effect, and the observed effect was a weakening, not strengthening of the biofilm. The chelant EDTA had negligible effect on the $S$. epidermidis biofilms.

An artifact of the concentrations of multivalent cations used is that the concentrations far exceeded the solubility of the corresponding metal hydroxide in water of neutral $\mathrm{pH}$. The solubility of aluminum (III) and iron (III) in water of neutral $\mathrm{pH}$ is in the $\mu \mathrm{M}$ range, far below the $0.2 \mathrm{M}$ or $0.02 \mathrm{M}$ concentrations added. While $\mathrm{Fe}(\mathrm{II})$ is soluble in this range, it also is susceptible to rapid oxidation to Fe (III) by oxygen diffusing in from the air. In addition, the formation of any metal hydroxides and even soluble hydroxo complexes would scavenge significant amounts of hydroxide ion from solution, possibly substantially decreasing the $\mathrm{pH}$. It is therefore possible that all the changes seen due to the addition of these ions were a result of a drop in $\mathrm{pH}$ rather than an effect of the ions themselves. The release of large numbers of protons, more mobile than the cations, could have affected EPS structure more significantly than the metal ions themselves. The differences in magnitude between the different cation treatments could have been as much due to the differing $\mathrm{pH}$ as to the differing cations. Control experiments with lowered $\mathrm{pH}$ values but no additional multivalent metal ions were not performed.

The biocidal or disinfectant based treatment of the $P$. aeruginosa biofilms all increased the peak strain values, although chlorine and urea did not produce statistically significant changes. Glutaraldehyde, QAC, and particularly ciprofloxacin produced significantly higher peak strain values and generally statistically significantly lower Burger parameter values. The ciprofloxacin, applied at about 10 times the minimum inhibitory concentration of $0.1 \mathrm{mg} 1^{-1}$, produced a creep curve that nearly matches that of a simple viscous fluid. No solid properties were part of the curve. The biocidal compounds applied to the $S$. epidermidis biofilm also produced an increase in the peak strain, but only rifampin, QAC and urea produced significant results. The rifampin dose was also ten times the MIC for this compound and organism (Zheng and Stewart 2002), but did not produce the radically different shape of creep curve observed with the ciprofloxacin and $P$. aeruginosa. The responses to the two antibiotics remain in many respects the most interesting. Unlike any of the other treatments, these were applied at doses well below the $\mu \mathrm{M}$ level, far too low to produce an effect purely due to chemical interactions. Thus, the effect was to cause changes in the biology occurring within the biofilm that led to disruption of the physical structure of the film.

The different mechanical properties of $P$. aeruginosa and $S$. epidermidis biofilms and their distinct responses to chemical treatments probably derive from the particular biochemical composition of the EPS of each bacterium. Alginate is thought to be a dominant polysaccharide in mucoid $P$. aeruginosa biofilms, although extracellular DNA is also found in the biofilm matrix of this microorganism (Steinberger and Holden 2005). The EPS of S. epidermidis reportedly contains a glucosamine polysaccharide, extracellular techoic acids, and unidentified proteins (Sadovskaya et al. 2005). Aggarwal and Hozalski (2010) measured the elastic modulus for S. epidermidis biofilm grown on a rotating disc reactor at around $1200 \mathrm{~Pa}$, and Aggarwal et al. (2010) measured the cohesive strengths of $S$. epidermidis and $P$. aeruginosa biofilms at $61-5840 \mathrm{~Pa}$ and $59-18,900 \mathrm{~Pa}$, respectively. The highly variable nature of the biofilm properties is illustrated by the range of values determined by these authors.

The creep curve data taken from the rotating disc viscometer with these biofilms contain a fair amount of variability, as evidenced by the need to analyze parameter values on a logarithmic scale in order to normalize the variance across replicates. It is reasonable, therefore, to ask whether tracking four parameters (five, if the peak strain value is included) to characterize the behavior is justified. Indeed, a review of the data in Table 1 shows that when one of the Burger parameters increased, they (generally speaking) all increased. However, as was shown in Figure 2, there are aspects of the creep curve particular to each of the four parameters; a simpler model would not be appropriate as it would fail to capture those details.

A second drawback to this approach is that the use of a parallel rotating disk does not produce a uniform shear stress or uniform strain across the entire biofilm surface. Stresses and strains near the center of the disc approach zero, and are at a maximum at the outer edge. For materials that do not exhibit a linear relationship between stress and either strain or strain 
rate, the results would not be very reproducible under different applied shear stresses. Nevertheless, as much consistency was applied as possible in performing the tests, and the results do allow comparison of the effects of different treatments.

\section{Conclusions}

(1) The Burger model for linear viscoelastic materials captured the behavior of biofilms during creep testing; (2) the two species of bacteria form biofilms with very different elastic and viscous properties; (3) treatments altered the material properties of the biofilms in some cases, but did not affect biofilms of the two species in the same way; (4) both biocidal and non-biocidal treatments can alter the material properties of biofilms, strengthening them in some cases and weakening them in others.

\section{Acknowledgements}

This work was supported by NIH award R01GM067245 and NSF award 0728621 , and by an award from the W.M. Keck Foundation.

\section{References}

Aggarwal S, Hozalski RM. 2010. Determination of biofilm mechanical properties from tensile tests performed using a micro-cantilever method. Biofouling 26:479-486.

Aggarwal S, Poppele EH, Hozalski RM. 2010. Development and testing of a novel microcantilever technique for measuring the cohesive strength of intact biofilms. Biotech Bioeng 105:924-934.

Anderl JN, Franklin MJ, Stewart PS. 2000. Role of antibiotic penetration limitation in Klebsiella pneumoniae biofilm resistance to ampicillin and ciprofloxacin. Antimicrob Agents Chemother 44:1818-1824.

Cense AW, Peeters EA, Gottenbos B, Baaijens FP, Nuijs AM, van Dongen ME. 2006. Mechanical properties and failure of Streptococcus mutans biofilms, studied using a microindentation device. J Microb Methods 67:463-472.

Chen X, Stewart PS. 1996. Chlorine penetration into artificial biofilm is limited by a reaction-diffusion interaction. Environ Sci Technol 30:2078-2083.

Chen X, Stewart PS. 2000. Biofilm removal caused by chemical treatments. Water Res 34:4229-4233.

Chen X, Stewart PS. 2002. Role of electrostatic interactions in cohesion of bacterial biofilms. Appl Microbiol Biotechnol 59:718-720.

Costerton JW, Stewart PS, Greenberg EP. 1999. Bacterial biofilms: a common cause of persistent infections. Science 284:1318-1322.

Habash M, Reid G. 1999. Microbial biofilms: their development and significance for medical device-related infections. J Clin Pharmacol 39:887-898.
Hohne DN, Younger JG, Solomon MJ. 2009. Flexible microfluidic device for mechanical property characterization of soft viscoelastic solids such as bacterial biofilms. Langmuir PMID: 19219968.

Klapper I, Rupp CJ, Cargo R, Purevdorj B, Stoodley P. 2002. A viscoelastic fluid description of bacterial biofilm material properties. Biotech Bioeng 80:289-296.

Korstgens V, Flemming HC, Wingender J, Dorchard W. 2001. Uniaxial compression measurement device for investigation of the stability of biofilms. J Microb Methods 46:9-17.

Lau PC, Dutcher JR, Beveridge TJ, Lan JS. 2009. Absolute quantitation of bacterial biofilm adhesion and viscoelasticity by microbead force spectroscopy. Biophys J 96:2935-2948.

Poppele EH, Hozalski RM. 2003. Micro-cantilever method for measuring the tensile strength of biofilms and microbial flocs. J Microbiol Methods 55:607-615.

Rupp ME, Ulphani JS, Fey PD, Mack D. 1999. Characterization of Staphylococcus epidermidis polysaccharide intercellular adhesin/hemagglutinin in the pathogenesis of intravascular catheter-associated infection in a rat model. Infect Immun 67:2656-2659.

Sadovskaya I, Vinogradov E, Flahaut SF, Kogan G, Jabbouri S. 2005. Extracellular carbohydrate-containing polymers of a model biofilm producing strain, Staphylococcus epidermidis RP62A. Infect Immun 73:3007-3017.

Silo-Suh L, Suh S, Sokol PA, Ohman DE. 2002. A simple alfalfa seedling infection model for Pseudomonas aeruginosa stains associated with cystic fibrosis shows AlfT (sigma-22) and RhlR contribute to pathogenesis. Proc Natl Acad Sci 99:15699-15704.

Steinberger RE, Holden PA. 2005. Extracellular DNA in single- and multiple-species unsaturated biofilms. Appl Environ Microbiol 71:5404-5410.

Stoodley P, Cargo R, Rupp CJ, Wilson S, Klapper I. 2002. Biofilm material properties as related to shear-induced deformation and detachment phenomena. J Ind Microbiol Biotechnol 29:361-367.

Towler BW, Rupp CJ, Cunningham AB, Stoodley P. 2003. Viscoelastic properties of a mixed culture biofilm from rheometer creep analysis. Biofouling 19:279-285.

Towler BW, Cunningham AB, Stoodley P, McKittrick L. 2007. A model of fluid-biofilm interaction using a Burger material law. Biotechnol Bioeng 96:259-271.

Van der Wende E, Characklis WG, Smith DB. 1989. Biofilms and bacterial drinking water quality. Water Res 23:13131322.

Walters III MC, Roe F, Bugnicourt A, Franklin MJ, Stewart PS. 2003. Contributions of antibiotic penetration, oxygen limitations, and low metabolic activity to tolerance of Pseudomonas aeruginosa biofilms to ciprofloxacin and tobramycin. Antimicrob Agents Chemother 47:317-323.

Wloka M, Rehage H, Flemming HC, Wingender J. 2005. Structure and rheological behavior of the extracellular polymeric substance network of mucoid Pseudomonas aeruginosa biofilms. Biofilms 2:275-283.

Zheng Z, Stewart PS. 2002. Penetration of rifampin through Staphylococcus epidermidis biofilms. Antimicrob Agents Chemother 46:900-903. 